\title{
Linked color imaging enhances endoscopic detection of sessile serrated adenoma/polyps
}

\section{(ㄷ)(1) $\odot$}

\author{
Authors

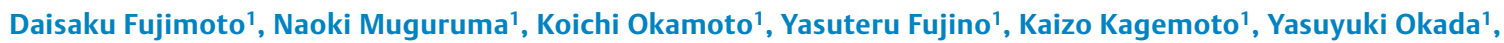 \\ Yoshifumi Takaoka', Yasuhiro Mitsui ${ }^{1}$, Shinji Kitamura', Tetsuo Kimura', Hiroshi Miyamoto', Yoshimi Bando², \\ Tomoko Sonoda ${ }^{3}$, Tetsuji Takayama ${ }^{1}$
}

\section{Institutions}

1 Department of Gastroenterology and Oncology, Institute of Biomedical Sciences, Tokushima University Graduate School, Tokushima, Japan.

2 Division of Pathology, Tokushima University Hospital, Tokushima, Japan.

3 Department of Public Health, Sapporo Medical University School of Medicine, Sapporo, Japan.

submitted 30.7.2017

accepted after revision 22.11.2017

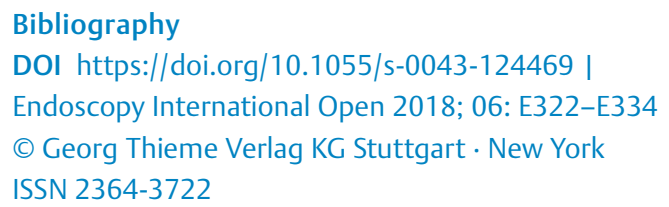

\section{Corresponding author}

Tetsuji Takayama, Department of Gastroenterology and Oncology, Institute of Biomedical Sciences Tokushima University Graduate School, 3-18-15, Kuramoto-cho, Tokushima city, 770-8503, Japan

Fax: +81-88-633-9235

takayama@tokushima-u.ac.jp

\section{ABSTRACT}

Background and study aims Although new image-enhanced endoscopy (IEE) technologies such as blue laser imaging (BLI), BLI-bright, and linked color imaging (LCI) have been developed, their utility for the detection of sessile serrated adenoma/polyps (SSA/Ps) is still unclear. This study aimed to evaluate the utility of BLI, BLI-bright, and $\mathrm{LCI}$ for SSA/P detection in still image examinations and in a prospective randomized controlled trial (RCT).
Patients and methods A group of 6 expert and non-expert endoscopists read 200 endoscopic still images containing SSA/P lesions using white light image (WLI), BLI, $\mathrm{BLI}-$ bright, and $\mathrm{LCl}$. Color differences were calculated using the color space method. A prospective RCT of tandem colonoscopy with WLI and $\mathrm{LCl}$ was performed. Patients with SSA/P and those with a history of SSA/P that had been endoscopically removed were enrolled and randomly allocated to WLI-LCI or LCI-WLI groups. Additional endoscopic detection rates for SSA/P were compared between the 2 groups.

Results $\mathrm{LCl}$ showed the highest SSA/P detection rate among the 4 modes for both expert and non-expert endoscopists. The detection rate with $\mathrm{LCl}$ for the 6 expert endoscopists (mean $98.3 \pm$ standard deviation $2.0 \%$ ) was significantly higher than that with WLI $(86.7 \pm 6.0 \%, P<0.01)$. Likewise, the detection rate with LCI for the 6 non-expert endoscopists $(92.3 \pm 2.9 \%)$ was significantly higher than that with WLI $(72.7 \pm 11.5 \%, P<0.01)$. The color difference of SSA/P with LCI was the highest among the 4 modes, and was significantly higher than with WLI (median 15.9, (interquartile range $13.7-20.6)$ vs. 10.2 , (7.6-14.2); $P<$ 0.0001 ). In the RCT, a total of 44 patients (WLI-LCI 22 vs. LCI-WLI 22) underwent colonoscopy. The additional detection rate for SSA/P in the second inspection in the WLI-LCI group $(21.6 \%, 8 / 37)$ was significantly higher than in the LCI-WLI group $(3.2 \%, 1 / 31 ; P=0.02)$. The small, flat, nonmucus and isochromatic SSA/Ps in the transverse colon were detected more frequently in the second inspection with $\mathrm{LCl}$.

Conclusions $\mathrm{LCl}$ was the most sensitive mode for SSA/P detection among WLI, BLI, BLI-bright, and $\mathrm{LCl}$ in the still image examinations. Our RCT strongly suggests that $\mathrm{LCl}$ is superior to conventional WLI for SSA/P detection during colonoscopy. UMIN000017599.

\section{Introduction}

Colorectal cancer (CRC) is 1 of the leading causes of cancerrelated deaths worldwide [1]. Endoscopic surveillance for premalignant polyps in the colorectum is an important strategy for reducing CRC-related mortality. Evidence has accumulated showing that endoscopic resection of adenoma reduces the development of CRCs [2]. However, several reports indicate that $25 \%$ to $28 \%$ of colorectal polyps are missed in colonoscopic ex- 
aminations $[3,4]$. In particular, colorectal polyps in the rightside colon are likely to be overlooked in colonoscopy [5]. In fact, many interval cancers have been reported, some of which were already at advanced or unresectable stages. Thus, overlooking pre-malignant or malignant lesions in colonoscopy remains a significant problem.

Recently, the serrated polyp-cancer sequence has received considerable attention. According to WHO criteria, serrated polyps are classified into 3 categories: hyperplastic polyp (HP), traditional serrated adenoma (TSA), and sessile serrated adenoma/polyp (SSA/P) [6]. Of these, SSA/P predominantly occurs in the right-side colon accompanied with B-RAF mutation and hypermethylation, and is considered to be a precursor lesion of microsatellite instability positive CRCs [7-9]. However, it is often difficult to detect SSA/P with conventional colonoscopy because the form of this lesion is typically flat and the color is similar to the background mucosa or more faded [7, 9]. Studies have shown that SSA/P is often overlooked and that it accounts for a significant proportion of interval cancers [10]. Thus, it is important to improve diagnostic detection sensitivity for SSA/P lesions. Image-enhanced endoscopy (IEE) technologies including narrow-band imaging (NBI), blue laser imaging (BLI), and BLI-bright have been newly developed [11-13]. A more recently developed IEE technology, linked color imaging (LCI), enhances reddish hues, which makes red areas appear redder and white areas appear whiter $[14,15]$. Dohi and associates reported that $\mathrm{LCl}$ could identify acute $\mathrm{H}$. pylori infection by enhancing the redness of the fundic gland mucosa [16]. Moreover, Sun and associates reported that $\mathrm{LCl}$ had higher diagnostic accuracy for gastrointestinal lesions including gastric cancers and colorectal cancers compared with BLI and white light (WLI) [17]. Therefore, it is expected that these technologies may improve the ability to diagnose SSA/Ps. Only 2 randomized trials have investigated the utility of NBI for the diagnosis of SSA/P $[18,19]$. However, neither of these trials showed improvement in the rate of detection for SSA/P (serrated lesions) during colonoscopy. Moreover, recent clinical trials showed that BLI and $\mathrm{LCl}$ improved the detection of colorectal polyps and adenomas [20, 21]. However, no clinical studies focusing on SSA/P detection with $\mathrm{BLI}, \mathrm{BLI}$-bright, or $\mathrm{LCI}$ have been reported to date. Therefore, in this study, we first tested the SSA/P detection ability of WLI, BLI, BLI-bright, and LCI by having endoscopists read multiple still images from each mode. Since LCl ultimately showed the highest detection rate for SSA/P among these modes, we next performed a prospective, randomized, controlled trial of tandem colonoscopy with WLI-LCI and LCI-WLI to investigate the superiority of $\mathrm{LCI}$ to WLI for detection of SSA/P.

\section{Patients and methods}

\section{Endoscopic equipment}

All examinations were carried out using a LASEREO laser endoscope system (Fujifilm Co., Tokyo, Japan) consisting of high-resolution colonoscopes (EC-L590ZW and EC-L600ZP), a light source (LL-4450), and a video processor (VP-4450HD). This system utilizes a semiconductor laser as the light source and has narrow-band light functions, which can produce 4 types of ima- ging including WLI, BLI, BLI-bright, and LCI [12-14]. BLI is performed with a combination of strong laser light at a wavelength of $410 \mathrm{~nm}$ and weak laser light at a wavelength of $450 \mathrm{~nm}$. BLIbright is set by controlling the power of the 2 lasers and an appropriate combination of WLI and $\mathrm{BLI}$. $\mathrm{LCl}$ is a novel image-enhancement technology based on images captured under conditions similar to those of BLI-bright $[14,15]$. It utilizes digital processing to enhance slight color differences in hue in the red region of the spectrum [22] ( Supplementary Fig.1). Each observation mode can be switched easily by pushing a button on the colonoscope manipulation handle.

\section{Detectability in still images}

Endoscopic Images of SSA/P lesions were taken with a distant view (3 to $5 \mathrm{~cm}$ distance) at the same location in each mode of WLI, BLI, BLI-bright and LCI. Fifty sets of images of SSA/P that were histologically proven were used. 6 expert and 6 non-expert endoscopists in Tokushima University Hospital, who had not been committed to photographing SSA/P still images, randomly examined a total of 200 still images (50 sets for each mode) that include SSA/P lesions. We defined an expert as an endoscopist who had performed more than 5,000 colonoscopies and 300 withdrawal colonoscopies with $\mathrm{LCl}$, and a non-expert as an endoscopist with less than 5,000 colonoscopies and 10 to 30 withdrawal colonoscopies with $\mathrm{LCI}$. The rate of correctly detecting the SSA/P lesion (detection rate) in each imaging mode was evaluated. In order to evaluate detectability, each image was scored on a 4-point scale; 4 =easily detectable; $3=$ reasonably detectable, 2 = barely detectable, and $1=$ undetectable. Individual image scores for each mode were then added to determine the detectability score. The kappa value was used to calculate the interobserver agreement in each mode.

\section{Color difference}

Color differences were calculated using the color space method [23].

\section{Clinical trial participants}

All patients were recruited at Tokushima University Hospital. For inclusion, patients were required to have had SSA/P or a history of endoscopic resection of SSA/P that had been proven histologically. Patients receiving anticoagulant therapy or having a history of surgical resection of the right colon and inflammatory bowel disease were excluded. All patients gave written informed consent for examination and treatment before the procedures.

\section{Study design of clinical trial}

The prospective randomized controlled trial of tandem colonoscopy was performed at Tokushima University Hospital. Patients underwent modified back-to-back colonoscopies with WLI and $\mathrm{LCl}$. Patients were randomly allocated to 1 of the 2 arms at a 1:1 ratio using a sealed envelope method: (1) WLI$\mathrm{LCl}$ group: first inspection with WLI followed by a second inspection with $\mathrm{LCl}$; (2) LCl-WLI group: first inspection with LCl followed by a second inspection with WLI. The protocol for this study was approved by the Institutional Review Board of the To- 
kushima University Hospital. The study was registered at the UMIN Clinical Trials Registry (UMIN000017599).

\section{Procedures}

Colonoscopy was performed with patients under conscious sedation with midazolam. After reaching the ileocecal region, in the WLI-LCI group, the colonoscope was withdrawn from the cecum to splenic flexure and reinserted into the cecum under WLI, and then withdrawn again to the splenic flexure and reinserted into the cecum under $\mathrm{LCI}$. In the $\mathrm{LCl}-\mathrm{WLI}$ group, the colonoscope was withdrawn from the cecum to the splenic flexure and reinserted into the cecum under $\mathrm{LCl}$, and then withdrawn again to the splenic flexure and reinserted into the cecum under WLI. Colonic mucosa was observed both backward and forward between the cecum and splenic flexure in each mode. Polyps detected during the first inspection were removed by snare or biopsied. Polyps detected during the second inspection were classified as additional polyps, and they were also removed by snare or biopsied.

For all polyps, the number, location, size, and morphology were recorded. The total time during both examinations, excluding the diagnostic time, as well as the polypectomy time and biopsy time were recorded using a stop watch. The quality of bowel preparation was assessed by modified Boston Bowel Preparation Scale (BBPS) [24], which includes a 4-point scoring system $(0-3)$ applied to each of the 2 segments of the right colon (ascending and transverse). Patients were excluded in cases of poor bowel preparation (mBBPS score $<4$ or 1 segment score $<2$ ). All procedures were performed by 5 endoscopists who had previously performed more than 1,000 colonoscopies and had at least 6 months of prior training performing observations with each of the 4 imaging modes.

\section{Outcomes}

The primary outcome of the study was the additional SSA/P detection rate for WLI- $\mathrm{LCl}$ (second inspection with $\mathrm{LCI}$ ) and $\mathrm{LCl}$ WLI (second inspection with WLI). The secondary outcomes were the positive detection rate per subject for additional SSA/ $\mathrm{P}$ lesions in the second inspection and the morphological features of additionally detected SSA/Ps.

\section{Sample size}

In our previous pilot study, the additional detection rates for SSA/P in LCI-WLI and WLI-LCI groups were $6.3 \%(1 / 16)$ and $33 \%$ (5/15), respectively. To detect a difference between the 2 groups with a power of $80 \%$ and $\alpha$-error of $5 \%$, the sample size needed was calculated to be 66 SSA/Ps. Since the patients with SSA/P had had an average number of 1.7 SSA/Ps in our hospital, we planned to enroll 43 patients considering an expected $10 \%$ dropout rate.

\section{Statistics}

The detection rate and detectability score for SSA/Ps were compared between the $\mathrm{WLI}$ group and the BLI, BLI-bright, or $\mathrm{LCI}$ group using Dunnett's test. Color differences were compared between the WLI group versus each of the other 3 groups using Wilcoxon signed-rank test with Bonferroni correction. Addi- tional detection rates for polyps, SSA/P, HP, and adenoma in the WLI-LCl and LCI-WLI groups were analyzed using Fisher's exact test.

Detailed methods of calculation of color difference, bowel cleansing, and histologic assessment are described in Supplementary methods.

\section{Results}

\section{Detection rate and detectability score for SSA/P in each mode}

To investigate the value of $\mathrm{BLI}, \mathrm{BLI}$-bright, and $\mathrm{LCl}$ for diagnosis of SSA/P, we first determined the detection rate for SSA/P among expert and non-expert endoscopists using 200 still pictures taken under each IEE mode ( $\triangleright$ Table 1$)$. The detection rate of SSA/P with LCI (mean 98.3 \pm standard deviation (SD) $2.0 \%$ ) was significantly higher than that with WLI $(86.7 \pm 6.0 \%$; $P<$ 0.01 ) for expert endoscopists. Conversely, the detection rate with BLI was significantly lower than with WLI $(71.0 \pm 5.2 \%$ vs. $86.7 \pm 6.0 ; P<0.01)$. There was no significant difference in rates between BLI-bright $(86.0 \pm 5.4 \%)$ and WLI for expert endoscopists. For non-expert endoscopists, the detection rates with $\mathrm{LCl}(92.3 \pm 2.9 \%)$ and BLI-bright $(86.7 \pm 7.0 \%)$ were significantly higher than with WLI $(72.7 \pm 11.5 \%) \quad(P<0.01$ and $P=0.01$ respectively). However, there was no significant difference in detection rates between BLI $(67.3 \pm 6.3 \%)$ and WLI. Thus, the detection rate for SSA/P with $\mathrm{LCI}$ was the highest among the 4 modes for both expert and non-expert endoscopists.

We also calculated detectability scores to reflect the ease of SSA/P diagnosis for each mode. The score with LCI (162.5 $\pm 17.1)$ was significantly higher than that with WLI (127.0 \pm 19.4 ) for expert endoscopists. However, there were no significant differences in scores between BLI $(118.5 \pm 16.2)$ and BLIbright $(130.7 \pm 19.3)$ versus WLI. For non-expert endoscopists, the scores with $\mathrm{LCl}(156.3 \pm 13.9)$ and BLI-bright $(142.5 \pm 12.8)$ were significantly higher than with WLI $(118.0 \pm 14.9)(P<0.01$ and $P=0.01$ respectively). There was no significant difference between the BLI $(121.2 \pm 10.1)$ and WLI groups. Thus, the detectability score with $\mathrm{LCl}$ was the highest among the 4 modes, and was significantly higher than that with WLI for both expert and non-expert endoscopists.

Among experts, the kappa values for WLI, BLI, BLI-bright and $\mathrm{LCl}$ were $0.659,0.771,0.768$ and 0.939 respectively, while among non-experts the values were $0.488,0.685,0.685$ and 0.805 respectively.

\section{Analysis of color difference}

To objectively evaluate SSA/P detection ability for the 4 modes, we calculated color differences between SSA/P and background mucosa in still images taken using each mode. Representative SSA/P lesions obtained from case 9 with each mode are shown in - Fig. 1a. The average color difference in WLI mode from the 4 ROIs set in the lesion and background mucosa was 21.3. Likewise, the color differences in BLI, BLI-bright and LCI mode were $35.1,30.7$, and 39.0, respectively. Thus, the color difference in $\mathrm{LCl}$ was the highest among the 4 modes. 
- Table 1 SSA/P detection rates and detectability scores in still images.

\begin{tabular}{|c|c|c|c|c|}
\hline & WLI & BLI & BLI-bright & $\mathbf{L C I}$ \\
\hline \multicolumn{5}{|c|}{ Detection rate (Mean \pm SD) } \\
\hline - Expert $(n=6)$ & $86.7 \pm 6.0$ & $71.0 \pm 5.2$ & $86.0 \pm 5.4$ & $98.3 \pm 2.0$ \\
\hline - Pvalue & & $<0.01$ & 0.99 & $<0.01$ \\
\hline - Non-expert $(n=6)$ & $72.7 \pm 11.5$ & $67.3 \pm 6.3$ & $86.7 \pm 7.0$ & $92.3 \pm 2.9$ \\
\hline - Pvalue & & 0.49 & 0.01 & $<0.01$ \\
\hline \multicolumn{5}{|c|}{ Detectability score (Mean \pm SD) } \\
\hline - Expert $(n=6)$ & $127.0 \pm 19.4$ & $118.5 \pm 16.2$ & $130.7 \pm 19.3$ & $162.5 \pm 17.1$ \\
\hline - Pvalue & & 0.92 & 0.97 & $<0.01$ \\
\hline - Non-expert $(n=6)$ & $118.0 \pm 14.9$ & $121.2 \pm 10.1$ & $142.5 \pm 12.8$ & $156.3 \pm 13.9$ \\
\hline - Pvalue & & 0.95 & 0.01 & $<0.01$ \\
\hline \multicolumn{5}{|c|}{$\begin{array}{l}\text { SSA/P, sessile serrated adenoma/polyp; WLI, white light image; BLI, blue light image; LCI, linked color image; } \\
\text { SD, standard deviation } \\
\text { The detection rate was expressed as the mean } \pm \text { SD of the rate (percentage) for each endoscopist ( } n=6) \text {. } \\
\text { The } P \text { values were calculated with Dunnett’s t test. }\end{array}$} \\
\hline
\end{tabular}

A total of 40 lesions were analyzed for color differences in the 4 modes ( $\mathbf{F i g} \mathbf{1} \mathbf{1 b}$ ). The color difference with LCl (median 15.9, interquartile range (IQR) 13.7-20.6) was significantly higher than that with WLI $(10.2,7.6-14.2$; $P<0.0001)$. The median color difference with BLI-bright (11.9, 8.6-15.1) was higher than with WLI despite no statistically significant difference $(P=0.07)$, while that with BLI $(10.7,7.8-15.0)$ was similar to that with WLI. These data clearly indicate that $\mathrm{LCl}$ is superior to WLI and other modes for detection of SSA/P.

In addition, the color differences on adenomas and HP in the 4 modes were analyzed ( $\triangleright$ Supplementary Fig. 2 ). The color difference on adenomas with $\mathrm{LCl}$ was highest among the 4 modes, similar to the findings on SSA/P. While there were no significant color difference on HP among the 4 modes.

\section{Clinical trial}

Based on data from the still image analysis, a prospective, randomized, controlled trial employing tandem colonoscopy was performed to investigate the superiority of LCI to WLI for detection of SSA/P.

\section{Characteristics of study population}

A flow diagram summarizing the disposition of patients is shown in Fig. 2. Between November 2014 and September 2016, 49 patients were assessed for eligibility and 3 were excluded. The remaining 46 patients were enrolled and randomly assigned to the WLI-LCl and LCI-WLI groups. 2 patients dropped out due to poor bowel preparation and 44 received colonoscopic examination. Colonoscopy reached the cecum in all patients and there were no procedure-related adverse events. A total of 22 patients in the WLI-LCI group and 22 patients in the $\mathrm{LCl}-\mathrm{WLI}$ groups were analyzed.

The demographic characteristics of participants are shown in $>$ Table2. There were no statistically significant differences in the male-to-female ratio, age, modified BBPS, or inspection time (withdrawal and insertion times) between the 2 groups.

\section{Additional SSA/P detection rate}

A total of 153 polyps were detected in the first inspection; 66 polyps in the WLI-LCI group and 87 in the LCI-WLI group ( $\downarrow$ Table 3 ). In the second inspection, a total of 20 polyps were detected; 16 in the WLI-LCI group and 4 in the LCI-WLI group. The rates of additional detection of polyps were $19.5 \%(16 / 82)$ in WLI-LCl and $4.4 \%(4 / 91)$ in LCI-WLI groups. The number of additionally detected polyps in the WLI-LCI group tended to be higher than that in the LCI-WLI group $(P=0.06)$.

Histological examination revealed that a total of 59 SSA/Ps were detected in the first inspection; 29 SSA/Ps in the WLI-LCI group and $30 \mathrm{SSA} / \mathrm{Ps}$ in the $\mathrm{LCl}-\mathrm{WLI}$ group. In the second inspection, a total of 9 SSA/Ps were additionally detected; 8 were detected in the WLI-LCI group but only 1 was detected in the $\mathrm{LCI}-\mathrm{WLI}$ group. The rates of additional detection of SSA/P in the WLI-LCI and LCI-WLI groups were $21.6 \%$ (8/37) and $3.2 \%$ $(1 / 31)$, respectively. The number of additionally detected SSA/ $\mathrm{P}$ in the WLI-LCI group was significantly higher than in the $\mathrm{LCl}$ WLI group $(P=0.02)$, indicating that this trial met the primary endpoint. Moreover, additional SSA/Ps were detected in the second inspection in 7 cases for the WLI-LCI group (1 SSA/P in 6 cases, and 2 SSA/Ps in 1 case), whereas only 1 SSA/P was detected in 1 case for the LCI-WLI group. The positive detection rate (per subject) for additional SSA/P lesions in the second inspection was significantly higher in the WLI-LCI group than in the LCI-WLI group ( $7 / 22$ vs. $1 / 22, P<0.02)$. As for additional detection of $\mathrm{HP}$ and adenomas in the second inspection, there were no significant differences in detection rates between the 2 groups, although the total number of those lesions might be small. 

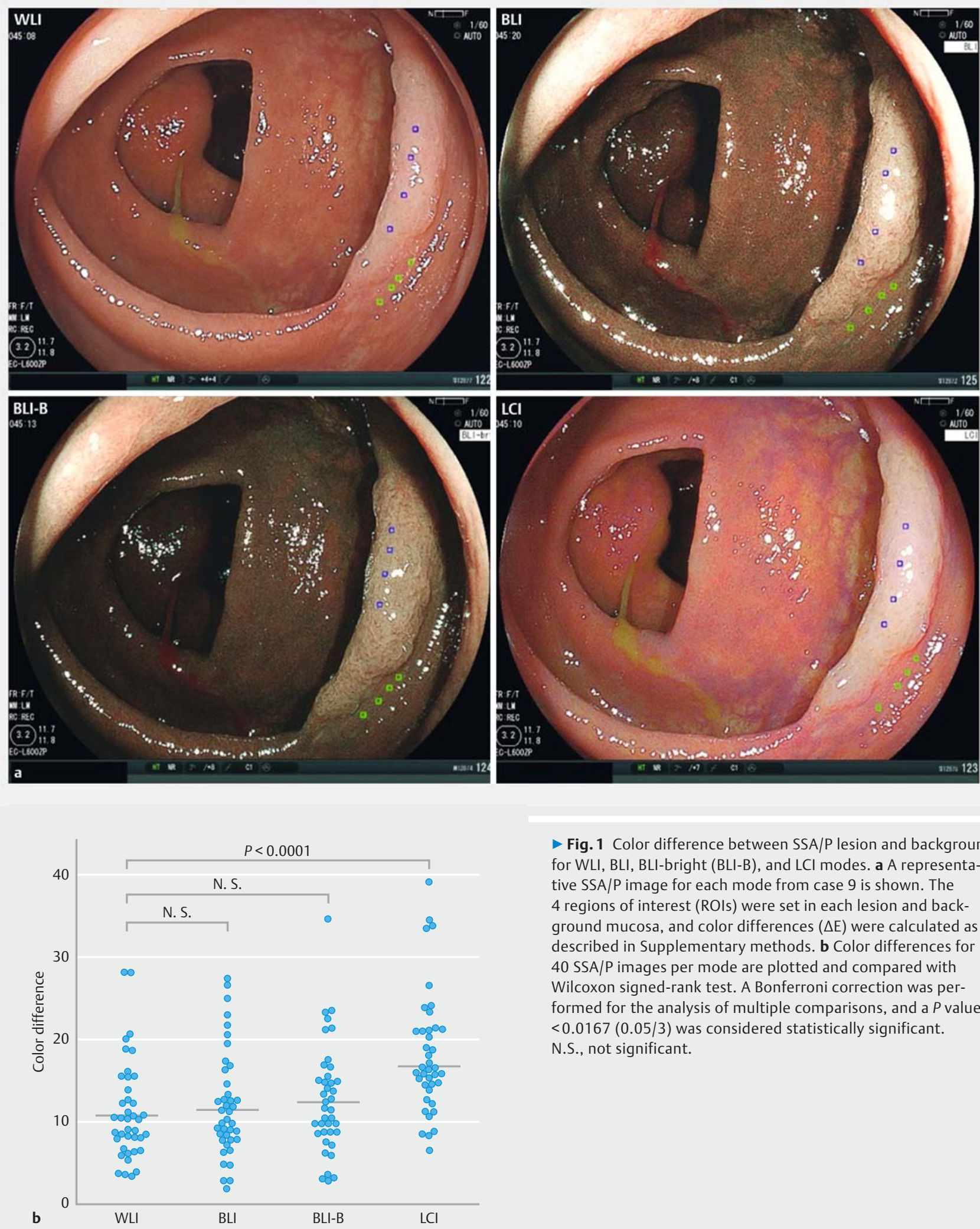

- Fig. 1 Color difference between SSA/P lesion and background for WLI, BLI, BLI-bright (BLI-B), and LCI modes. a A representative SSA/P image for each mode from case 9 is shown. The 4 regions of interest (ROIs) were set in each lesion and background mucosa, and color differences $(\Delta \mathrm{E})$ were calculated as described in Supplementary methods. b Color differences for $40 \mathrm{SSA} / \mathrm{P}$ images per mode are plotted and compared with Wilcoxon signed-rank test. A Bonferroni correction was performed for the analysis of multiple comparisons, and a $P$ value $<0.0167(0.05 / 3)$ was considered statistically significant. N.S., not significant. 


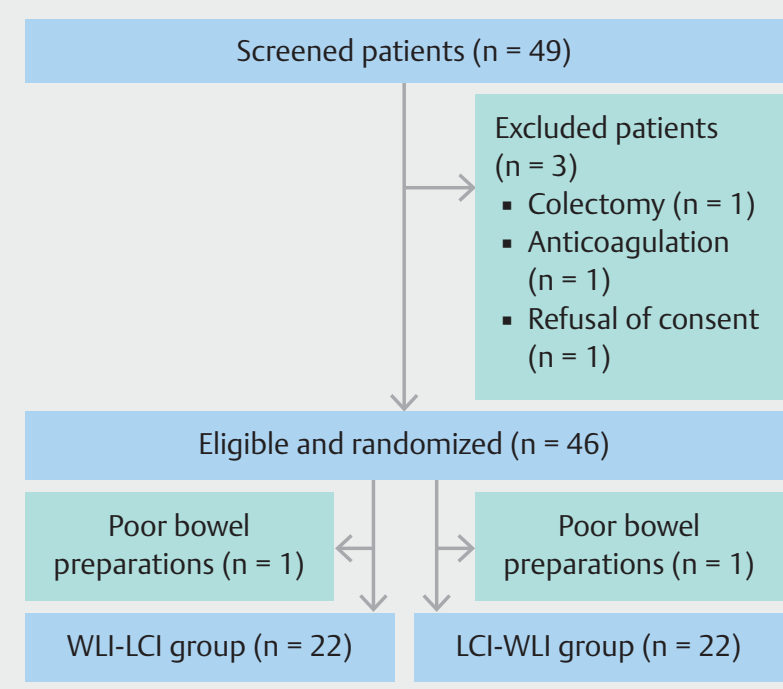

- Fig. 2 Flowchart of the randomized controlled trial.

\section{Features of additionally detected SSA/Ps in the WLI-LCI group}

To clarify the clinical and morphological features of additionally detected SSA/Ps in the second inspection by $\mathrm{LCl}$, a comparison should be made between the WLI-LCl and LCI-WLI groups. However, because only a single SSA/P was detected in the latter group, we compared SSA/Ps detected by WLI in the first inspection and second inspection by $\mathrm{LCl}$ in the WLI-LCl group ( $\triangleright$ Table 4). The rates of SSA/Ps smaller than $10 \mathrm{~mm}$, flat SSA/Ps, SSA/Ps without mucus, and isochromatic SSA/Ps were higher in the second inspection than in the first inspection, although the differences were not statistically significant between the 2 groups. In terms of the location of SSA/P lesions, interestingly, the frequency of SSA/Ps in the transverse colon was significantly higher in the second inspection than in the first inspection ( $P$ $=0.03$ ).

\section{Discussion}

In our initial study with endoscopic still images, we demonstrated that $\mathrm{LCl}$ is the most sensitive mode for colonoscopic detection of SSA/Ps as compared with WLI, BLI, and BLI-bright for both expert and non-expert endoscopists. Moreover, this result was objectively supported by evaluating color differences between SSA/P and background mucosa; the average color difference was by far the highest in $\mathrm{LCl}$ mode. In addition, in the subsequent randomized controlled trial, we clearly showed that $\mathrm{LCl}$ is superior to conventional WLI with a significantly greater ability to detect SSA/P during colonoscopy. These results strongly suggest that endoscopic observation utilizing $\mathrm{LCl}$ mode improves the detection and diagnosis of SSA/Ps that may sometimes be overlooked with conventional WLI observation. Until now, no prospective study has investigated the utility of $\mathrm{LCl}$ for the detection of SSA/Ps. This is the first study to demonstrate the value of $\mathrm{LCl}$ for detecting SSA/P. 1 of this study's strengths is that it employed 2 different analyses (i. e., still image analysis with color difference evaluation and a subsequent randomized clinical trial), which provided compatible results.

In the analysis of still images, the detection rates for SSA/P in conventional WLI mode were $86.7 \%$ among expert endoscopists and $72.7 \%$ among non-expert endoscopists; a $14.0 \%$ difference between the 2 groups. With $\mathrm{LCl}$, the rates were

- Table 2 Patient characteristics in WLI-LCl and LCI-WLI groups.

\begin{tabular}{|c|c|c|c|}
\hline & WLI-LCI (n= 22) & LCI-WLI (n= 22) & $P$ value \\
\hline Male (n, \%) & $8(38)$ & $12(57)$ & $0.26^{1}$ \\
\hline Age (mean $\pm S D$, year) & $61.1 \pm 9.3$ & $65.9 \pm 10.6$ & $0.20^{2}$ \\
\hline Modified BBPS score (median, range) & $5(4-6)$ & $5(4-6)$ & $0.60^{3}$ \\
\hline \multicolumn{4}{|l|}{ Inspection time (mean $\pm S D$, second) } \\
\hline \multicolumn{4}{|l|}{ First inspection } \\
\hline - Withdrawal time & $334 \pm 118$ & $314 \pm 83$ & $0.63^{2}$ \\
\hline - Insertion time & $231 \pm 102$ & $190 \pm 86$ & $0.28^{2}$ \\
\hline - Total & $565 \pm 182$ & $504 \pm 131$ & $0.34^{2}$ \\
\hline \multicolumn{4}{|l|}{ Second inspection } \\
\hline - Withdrawal time & $272 \pm 92$ & $254 \pm 71$ & $0.58^{2}$ \\
\hline - Insertion time & $190 \pm 66$ & $146 \pm 72$ & $0.12^{2}$ \\
\hline - Total & $461 \pm 140$ & $399 \pm 118$ & $0.24^{2}$ \\
\hline \multicolumn{4}{|c|}{$\begin{array}{l}\text { BBPS, Boston bowel preparation scale; SD, standard deviation. } \\
{ }^{1} \text { The } P \text { value was calculated with chi-square test. } \\
{ }^{2} \text { The } P \text { value was calculated with Student's } t \text { test. } \\
{ }^{3} \text { The } P \text { value was calculated with Mann-Whitney U test. }\end{array}$} \\
\hline
\end{tabular}


- Table 3 Additional polyp detection rates in WLI-LCl and LCI-WLI groups.

\begin{tabular}{|c|c|c|c|}
\hline & WLI-LCI $(n=22)$ & LCI-WLI $(n=22)$ & $P$ value \\
\hline \multicolumn{4}{|l|}{ All polyps } \\
\hline - First inspection & 66 & 87 & \\
\hline - Second inspection & 16 & 4 & \\
\hline - Additional detection rate & $19.5(16 / 82)$ & $4.4(4 / 91)$ & 0.06 \\
\hline \multicolumn{4}{|l|}{$\mathrm{SSA} / \mathrm{P}$} \\
\hline - First inspection & 29 & 30 & \\
\hline - Second inspection & 8 & 1 & \\
\hline - Additional detection rate & $21.6(8 / 37)$ & $3.2(1 / 31)$ & 0.02 \\
\hline \multicolumn{4}{|l|}{ HP } \\
\hline - First inspection & 8 & 6 & \\
\hline - Second inspection & 5 & 1 & \\
\hline - Additional detection rate & $38.5(5 / 13)$ & $14.3(1 / 7)$ & 0.54 \\
\hline \multicolumn{4}{|l|}{ Adenomas } \\
\hline - First inspection & 29 & 51 & \\
\hline - Second inspection & 3 & 2 & \\
\hline - Additional detection rate & $9.4(3 / 32)$ & $3.8(2 / 53)$ & 0.64 \\
\hline
\end{tabular}

- Table 4 Features of additionally detected SSA/Ps in WLI-LCI group

\begin{tabular}{|c|c|c|c|}
\hline & WLI (first inspection) $(n=29)$ & LCI (second inspection) $(\mathrm{n}=8)$ & $P$ value \\
\hline Size $(\mathrm{mm})$ & & & 0.38 \\
\hline . $<10$ & $14(48.3 \%)$ & $5(62.5 \%)$ & \\
\hline " $\geq 10$ & $15(51.7 \%)$ & $3(37.5 \%)$ & \\
\hline Morphology & & & 0.55 \\
\hline - Protrude & $9(31.0 \%)$ & $1(12.5 \%)$ & \\
\hline - Flat & $20(69.0 \%)$ & $7(87.5 \%)$ & \\
\hline Mucus & & & 0.22 \\
\hline - None & $5(17.2 \%)$ & $3(37.5 \%)$ & \\
\hline - Some & $24(82.8 \%)$ & $5(62.5 \%)$ & \\
\hline Color & & & 0.49 \\
\hline - Fading & $15(51.7 \%)$ & $3(37.5 \%)$ & \\
\hline - Same & $12(41.4 \%)$ & $5(62.5 \%)$ & \\
\hline - Reddish & $2(6.9 \%)$ & $0(0.0 \%)$ & \\
\hline Location & & & 0.03 \\
\hline - Cecum & $9(31.0 \%)$ & $1(12.5 \%)$ & \\
\hline - Ascending & $13(44.8 \%)$ & $1(12.5 \%)$ & \\
\hline - Transverse & $7(24.1 \%)$ & $6(75.0 \%)$ & \\
\hline
\end{tabular}


$11.6 \%$ and $19.6 \%$ higher among expert and non-expert endoscopists, respectively. Thus, while both groups of endoscopists received significant benefit from $\mathrm{LCl}$, the benefit was even greater for non-expert endoscopists. Moreover, BLI-bright also increased the detection rates for non-experts by $14.0 \%$ but did not have an impact on the rates for experts. Similar results were obtained in the analysis of detectability scores for experts and non-experts. It is possible that expert endoscopists did not receive any benefit from BLI-bright because their rate of SSA/P detection with WLI (86.7\%) was fairly high compared with non-experts (72.7\%). Moreover, expert endoscopists might be more skilled in detecting SSA/P by utilizing other factors such as morphology, presence of mucus cap, etc. Furthermore, the color difference with LCI (median 15.9, IQR 13.7-20.6) was much higher, and the difference with BLI-bright (11.9, 8.615.1) was slightly higher, as compared with those for WLI $(10.2,7.6-14.2)$ and $\mathrm{BLI}(10.7,7.8-15.0)$. These results for color difference also support the results obtained with respect to high detection rates and high detectability scores with $\mathrm{LCl}$. Moreover, our results are also supported by a recent study indicating that visibility scores for colorectal nongranular flat lesions in $\mathrm{LCl}$ still images were higher than those in WLI still images [25].

Our randomized controlled trial showed that the rate of additional detection of SSA/P in the WLI-LCI group $(21.6 \%)$ was significantly higher than that in $\mathrm{LCl}-\mathrm{WLI}$ group $(3.2 \%, P=0.02)$. This indicates that our trial met the primary endpoint, which was to demonstrate superiority of LCI to WLI for SSA/P detection. In this trial, HP and adenomas were also additionally detected in the second inspection for the WLI-LCI and LCI-WLI groups. The rates of additional detection of HP and adenomas in the WLI-LCI group were also higher than in the LCI-WLI group. Since these polyps are easily detectable due to their protruding morphology, detection by WLI may be sufficient [26]. The value of $\mathrm{LCl}$ for the detection of these polyps should be further investigated.

The analysis of features of additionally detected SSA/P in the second inspection with $\mathrm{LCI}$ revealed that the SSA/Ps with a small diameter $(<10 \mathrm{~mm})$, with flat shape, without a mucus cap, and with the same color were more frequently detected despite the lack of a statistically significant difference. This result is quite reasonable and also consistent with previous reports that the presence of a mucus cap is an important finding for SSA/P detection [27]. Moreover, SSA/P lesions detected in the second inspection with $\mathrm{LCl}$ were predominantly located in the transverse colon than in the first inspection with WLI. This result may be partly explained by the fact that the transverse colon is not fixed to the retroperitoneum like the sigmoid colon, and therefore, lesions in the transverse colon are sometimes difficult to visualize squarely. In fact, it is reported that polyps in the transverse colon and sigmoid colon are most likely to be missed during colonoscopy [28]. These results suggested that SSA/P lesions in the transverse colon, particularly when small, flat, mucus cap-free, and of the same color are likely to be overlooked during colonoscopy with WLI.

Limitations of this study are that the clinical trial was performed at a single institution and the number of enrolled pa- tients was relatively small. Another limitation is that our trial recruited only patients with SSA/P and a history of endoscopic resection of SSA/P. Therefore, a multicenter, larger-scale trial recruiting a more general patient population to compare SSA/P detection with $\mathrm{LCl}$ and WLI could strengthen our results.

\section{Conclusion}

Our still image examinations suggested that $\mathrm{LCl}$ is the most sensitive mode for SSA/P detection during colonoscopy as compared with WLI, BLI, and BLI-bright modes. RCT data demonstrated that the rate of additional detection of SSA/P with WLI$\mathrm{LCl}$ was significantly higher than with $\mathrm{LCl}-\mathrm{WLI}$. Thus, our data strongly suggest that $\mathrm{LCl}$ enhances the detection of SSA/P in colonoscopy.

\section{Acknowledgement}

The authors are grateful to Drs. Shunto J (Shunto clinic, Tokushima, Japan) and Kataoka K (Kataoka Clinic, Tokushima, Japan) for referring SSA/P patients to our hospital. We are also grateful to Drs, Okada R, Kawaguchi T, Kurihara K, Matsumoto R, Noda K, Yamamoto K, Fukuya A (Tokushima University Hospital, Tokushima, Japan) for their help in the still image examinations.

\section{Competing interests}

This study was supported, in part, by a collaborative research grant between Tokushima University and Fujifilm Co. Dr. Takayama received a research grant from Fujifilm Co. The financial sponsor was not involved in the design of the study, analysis and interpretation of the data, or the preparation of the manuscript.

\section{References}

[1] Siegel RL, Miller KD, Jemal A. Cancer statistics, 2016. CA Cancer J Clin 2016; 66: 7-30

[2] Zauber AG, Winawer SJ, O'Brien MJ et al. Colonoscopic polypectomy and long-term prevention of colorectal-cancer deaths. N Engl J Med 2012; 366: 687-696

[3] Heresbach D, Barrioz T, Lapalus MG et al. Miss rate for colorectal neoplastic polyps: a prospective multicenter study of back-to-back video colonoscopies. Endoscopy 2008; 40: 284-290

[4] Leufkens AM, van Oijen MG, Vleggaar FP et al. Factors influencing the miss rate of polyps in a back-to-back colonoscopy study. Endoscopy 2012; 44: 470-475

[5] Singh H, Nugent Z, Demers AA et al. The reduction in colorectal cancer mortality after colonoscopy varies by site of the cancer. Gastroenterology 2010; 139: $1128-1137$

[6] Snover DC, Ahnen D, Burt R et al. Serrated polyps of the colon and rectum and serrated polyposis WHO classification of tumours of the digestive system. Lyon (France): IARC; 2010: 160-165

[7] Higuchi T, Sugihara K, Jass JR. Demographic and pathological characteristics of serrated polyps of colorectum. Histopathology 2005; 47 : $32-40$ 
[8] Kambara T, Simms LA, Whitehall VL et al. BRAF mutation is associated with DNA methylation in serrated polyps and cancers of the colorectum. Gut 2004; 53: $1137-1144$

[9] Leggett B, Whitehall V. Role of the serrated pathway in colorectal cancer pathogenesis. Gastroenterology 2010; 138: 2088-2100

[10] IJspeert JE, van Doorn SC, van der Brug YM et al. The proximal serrated polyp detection rate is an easy-to-measure proxy for the detection rate of clinically relevant serrated polyps. Gastrointest Endosc 2015; 82: $870-877$

[11] Hazewinkel Y, Dekker E. Colonoscopy: basic principles and novel techniques. Nat Rev Gastroenterol Hepatol 2011; 8: 554 - 564

[12] Yoshida N, Yagi N, Inada Y et al. The ability of a novel blue laser imaging system for the diagnosis of colorectal polyps. Dig Endosc 2014; 26: $250-258$

[13] Kaneko K, Oono Y, Yano T et al. Effect of novel bright image enhanced endoscopy using blue laser imaging (BLI). Endosc Int Open 2014; 2: E212- 219

[14] Okada M, Sakamoto H, Takezawa T et al. Laterally spreading tumor of the rectum delineated by linked color imaging technology. Clin Endosc 2016; 49: $207-208$

[15] Fukuda H, Miura Y, Hayashi Y et al. Linked color imaging technology facilitates early detection of flat gastric cancers. Clin J Gastroenterol 2015; 8: 385-389

[16] Dohi O, Yagi N, Onozawa Y et al. Linked color imaging improves endoscopic diagnosis of active Helicobacter pylori infection. Endosc Int Open 2016; 4: E800-805

[17] Sun X, Dong T, Bi Y et al. Linked color imaging for improving the endoscopic diagnosis accuracy: a pilot study. Sci Rep 2016; 6: 33473

[18] Hazewinkel Y, Tytgat KM, van Leerdam ME et al. Narrow-band imaging for the detection of polyps in patients with serrated polyposis syndrome: a multicenter, randomized, back-to-back trial. Gastrointest Endosc 2015; 81: $531-538$

[19] Rex DK, Clodfelter R, Rahmani F et al. Narrow-band imaging versus white light for the detection of proximal colon serrated lesions: a randomized, controlled trial. Gastrointest Endosc 2016; 83: 166-171
[20] Ikematsu H, Sakamoto T, Togashi K et al. Detectability of colorectal neoplastic lesions using a novel endoscopic system with blue laser imaging: a multicenter randomized controlled trial. Gastrointest Endosc 2017; 86: $386-394$

[21] Min M, Deng P, Zhang W et al. Comparison of linked color imaging and white-light colonoscopy for detection of colorectal polyps: a multicenter, randomized, crossover trial. Gastrointest Endosc 2017; 86: $724-730$

[22] Kitagawa Y, Hara T, Ikebe D et al. Magnified endoscopic observation of small depressed gastric lesions using linked color imaging with indigo carmine dye. Endoscopy 2018; 50: 142 - 147

[23] Kuehni RG. Color-tolerance data and the tentative CIE $1976 \mathrm{~L}$ a b formula. J Opt Soc Am 1976; 66: 497 - 500

[24] Calderwood AH, Jacobson BC. Comprehensive validation of the Boston Bowel Preparation scale. Gastrointest Endosc 2010; 72: 686-692

[25] Suzuki T, Hara T, Kitagawa Y et al. Linked-color imaging improves endoscopic visibility of colorectal nongranular flat lesions. Gastrointest Endosc 2017; 86: 692 - 697 [Epub ahead of print]

[26] Singh R, Cheong KL, Zorron ChengTao Pu L et al. Multicenter randomised controlled trial comparing the high definition white light endoscopy and the bright narrow band imaging for colon polyps. World J Gastrointest Endosc 2017; 9: 273 -281

[27] Uraoka T, Higashi R, Horii ] et al. Prospective evaluation of endoscopic criteria characteristic of sessile serrated adenomas/polyps. J Gastroenterol 2015; 50: $555-563$

[28] Postic G, Lewin D, Bickerstaff C et al. Colonoscopic miss rates determined by direct comparison of colonoscopy with colon resection specimens. Am J Gastroenterol 2002; 97: 3182 - 3185

[29] Ball AJ, Johal SS, Riley SA. Position change during colonoscope withdrawal increases polyp and adenoma detection in the right but not in the left side of the colon: results of a randomized controlled trial. Gastrointest Endosc 2015; 82: 488 - 494

[30] Schlemper RJ, Riddell RH, Kato Y et al. The Vienna classification of gastrointestinal epithelial neoplasia. Gut 2000; 47: 251 - 255 


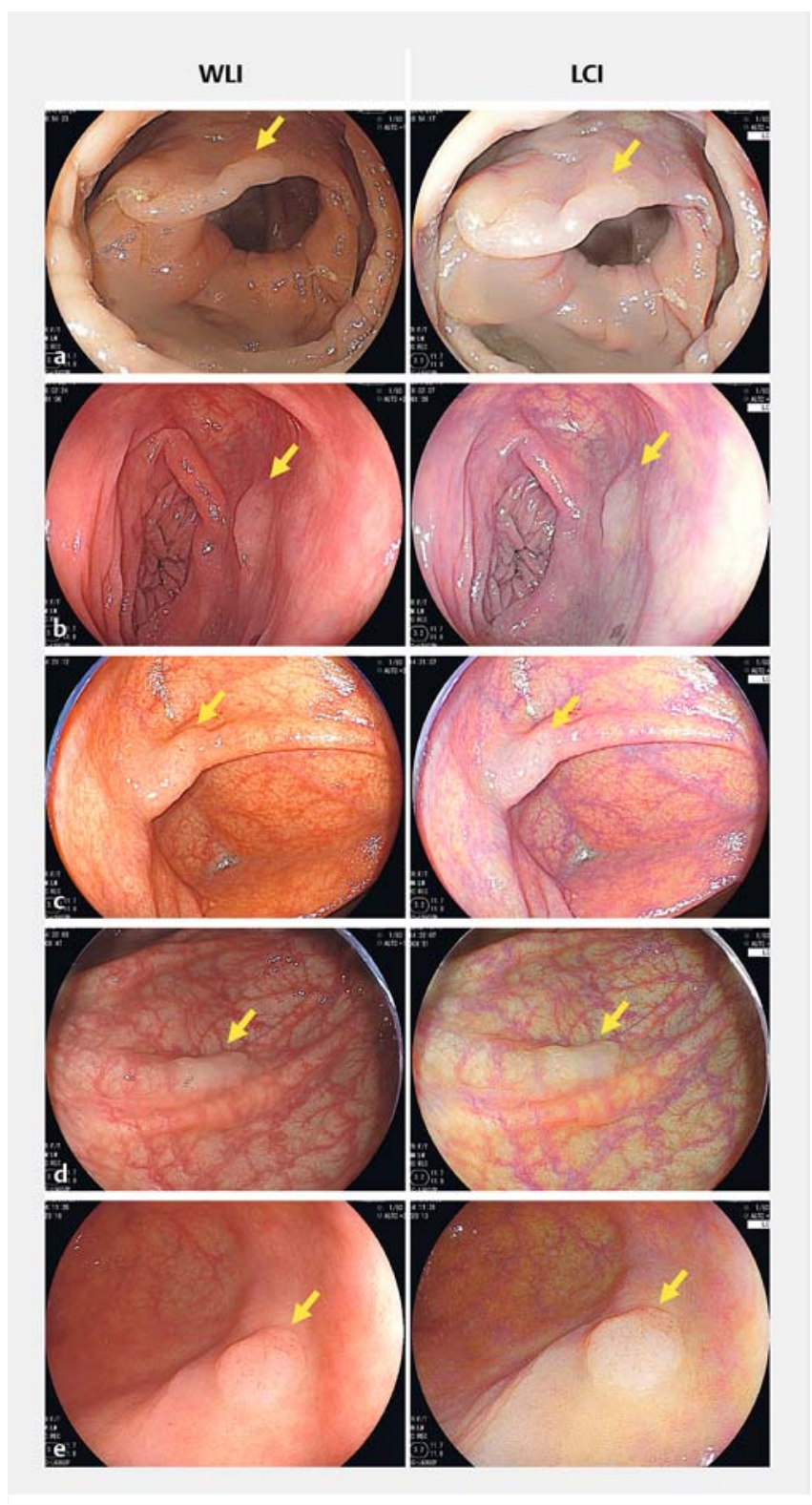

- Supplementary Fig. 1 Endoscopic images of SSA/P taken under linked color imaging (LCI) and white light (WLI). Various endoscopic still images of SSA/Ps with $\mathrm{LCl}$ and WLI are shown in a to e. Slight color differences were enhanced in LCI images. a SSA/P in ascending colon from 67-year-old man. b SSA/P in transverse coIon from 41-year-old woman. c SSA/P in cecum from 73-year-old woman. d SSA/P in transverse colon from 52-year-old woman. e SSA/P in cecum from 74-year-old woman. 

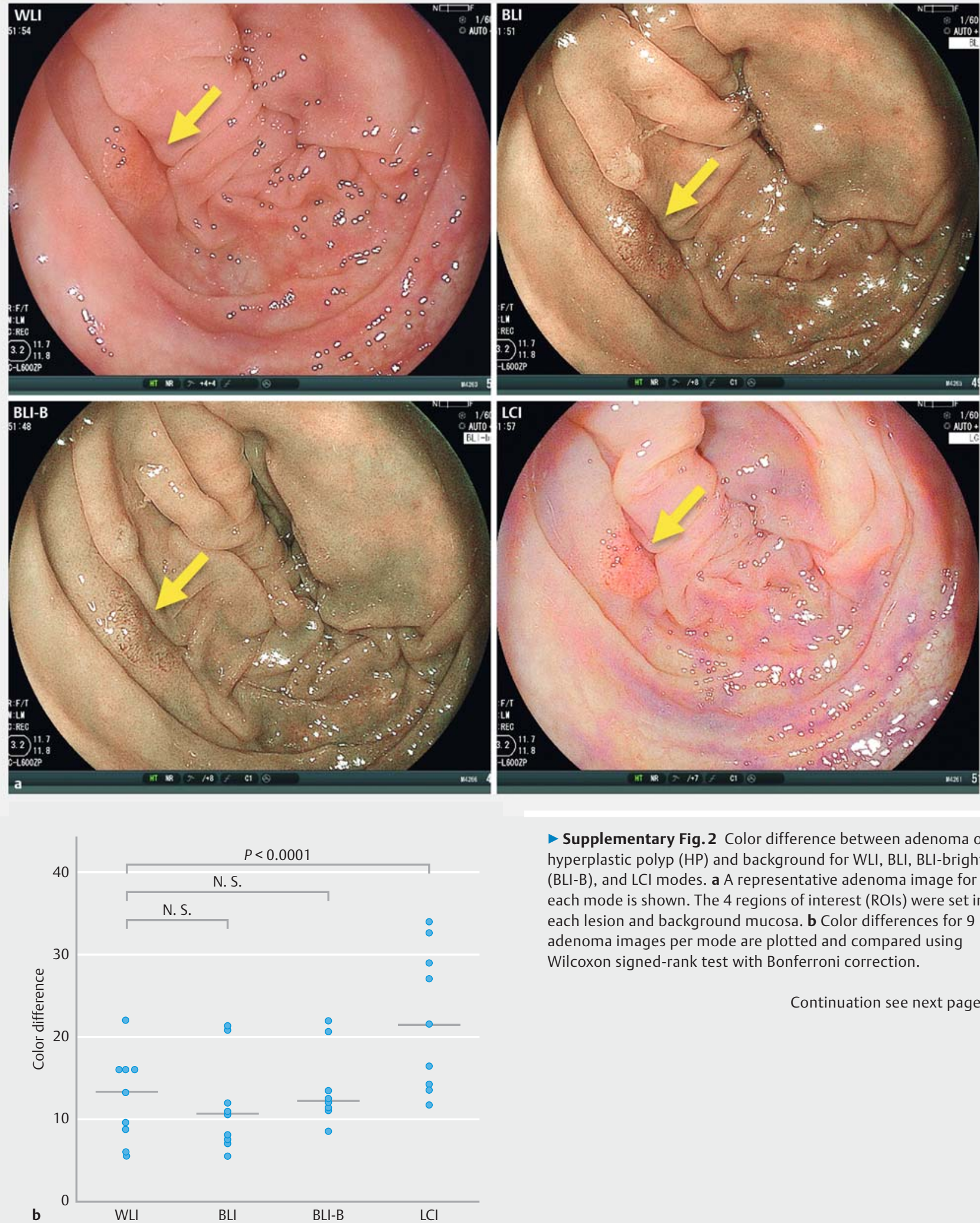

- Supplementary Fig. 2 Color difference between adenoma or hyperplastic polyp (HP) and background for WLI, BLI, BLI-bright (BLI-B), and $\mathrm{LCl}$ modes. a $\mathrm{A}$ representative adenoma image for each mode is shown. The 4 regions of interest (ROIs) were set in each lesion and background mucosa. $\mathbf{b}$ Color differences for 9 adenoma images per mode are plotted and compared using Wilcoxon signed-rank test with Bonferroni correction.

Continuation see next page. 

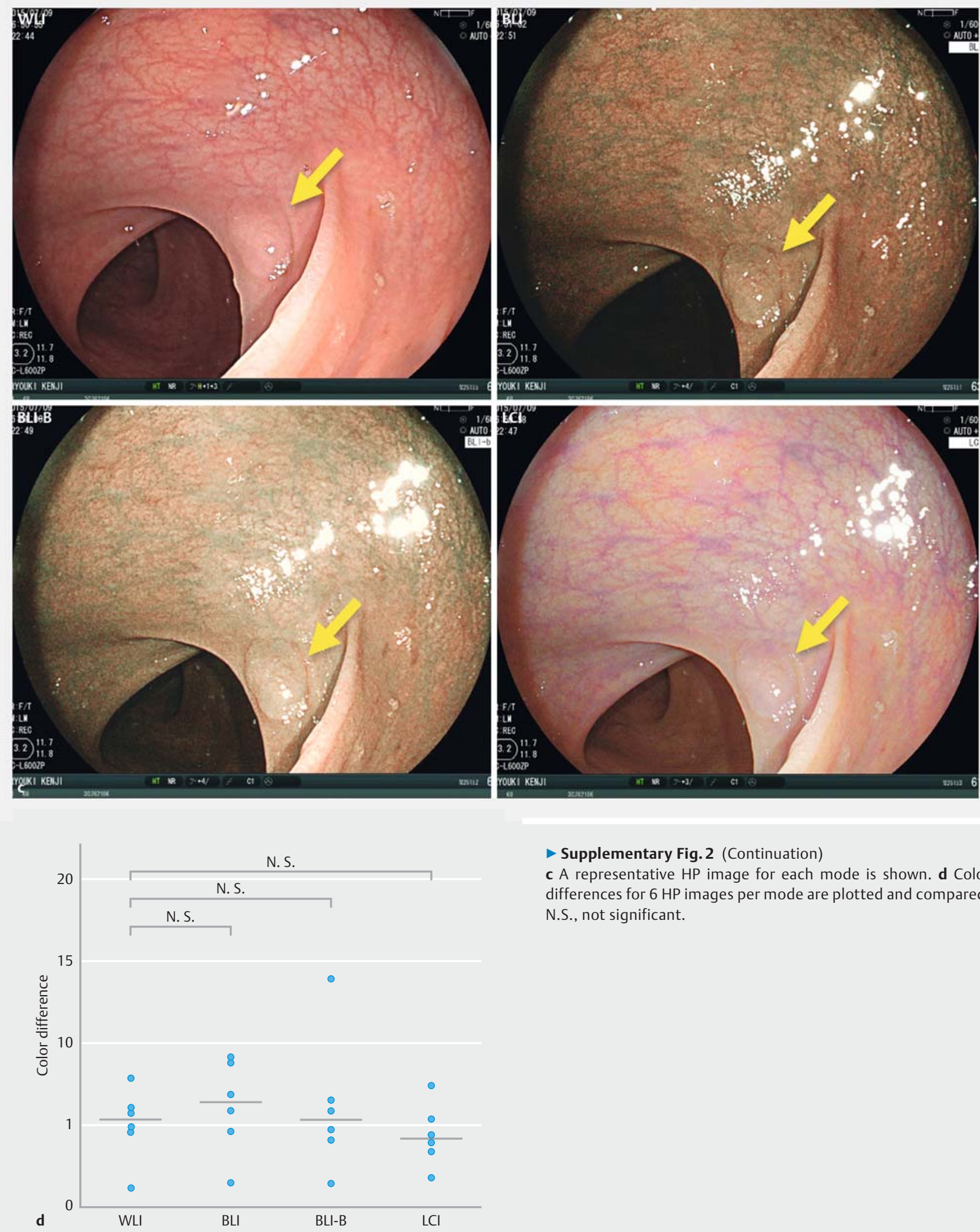

- Supplementary Fig. 2 (Continuation)

c A representative HP image for each mode is shown. d Color differences for $6 \mathrm{HP}$ images per mode are plotted and compared. N.S., not significant. 


\section{Supplementary methods}

\section{Calculation of color difference}

Color differences $(\Delta \mathrm{E})$ between SSA/P lesions and background mucosae were calculated according to the color space method specified by Commission internationale de l'éclairage (CIE) in 1976 [23]. The color of an arbitrary point is defined by a darklight value ( $L^{*}$ axis), red-green value ( $a^{*}$ axis), and yellow-blue value ( $b^{*}$ axis) in a 3-dimensional color space. The $\Delta E^{*}$ ab between color $1\left(L^{*} 1, a^{*} 1, b^{*} 1\right)$ and color $2\left(L^{*} 2, a^{*} 2, b^{*} 2\right)$ was calculated according to the following formula;

$$
\Delta \mathrm{E} * \mathrm{ab}=\sqrt{\left(\mathrm{L}_{1}^{*}-\mathrm{L}_{2}^{*}\right)^{2}+\left(\mathrm{a}_{1}^{*}-\mathrm{a}_{2}^{*}\right)^{2}+\left(\mathrm{b}_{1}^{*}-\mathrm{b}_{2}^{*}\right)^{2}}
$$

4 regions of interest (ROIs) (measuring $9 \times 9$ pixels) were assigned for SSA/P lesions and adjacent background mucosae in each still image. Each ROI was set roughly in the same regions in all 4 detection modes, sheering away foreign substances and halation. All ROIs were set by an expert endoscopist (N.M.). The color space (CIE L*, a*, b*) in each ROI of SSA/P and background mucosa was determined, and the $\triangle E$ was calculated using the mean value of the color space $\left(C I E L^{*}, a^{*}, b^{*}\right)$ in 4 ROIs.

\section{Bowel cleansing for colonoscopy}

On the day prior to colonoscopy, patients received $24 \mathrm{mg}$ sennoside (Pursennid; Novartis Pharma, Tokyo, Japan) as preparatory medication. The following morning, patients were prepared by ingesting $2 \mathrm{~L}$ polyethylene glycol solution containing ascorbic acid (Moviprep; EA Pharma, Tokyo, Japan) with an additional $2 \mathrm{~L}$ of clear liquids.

\section{Body position during colonoscopy}

To minimize the influence of position change during the observation [29], the ascending colon and transverse colon were examined thoroughly in the supine position during the first and second inspections.

\section{Histologic assessment}

All resected and biopsy specimens were retrieved, immediately fixed in $10 \%$ buffered formalin solution, and examined histologically by hematoxylin and eosin staining. A single pathologist specializing in Gl pathology reviewed all tissues. Specimens were classified according to the Vienna criteria for GI epithelial neoplasia [30]. Serrated polyps were classified according to the WHO 2010 classification of tumors of the digestive system [6]. When it was not possible to differentiate between SSA/P and hyperplastic polyp, the lesion was classified as a hyperplastic polyp. 